\title{
Effects of structural tests on aircraft safety
}

\author{
Erdem Acar ${ }^{1}$ \\ TOBB University of Economics and Technology, Sögütözü, Ankara 06560, Turkey \\ Raphael T. Haftka ${ }^{2}$, Nam-Ho Kim ${ }^{3}$, and Deepti Buchi ${ }^{4}$ \\ University of Florida, Gainesville, FL 32611, USA
}

\begin{abstract}
This paper investigates the effects of structural tests on aircraft safety. In particular, the paper focuses on the effect of the number of coupon tests and structural element tests on the final distribution of failure stress. The mean failure stress is assumed to be predicted by a failure criterion (e.g. Tsai-Wu), and the initial distribution of this mean failure stress reflects the uncertainty in the analysis procedure that uses coupon test data to predict structural failure. In addition to the uncertainty in the mean failure stress, there is also uncertainty in its variability due to the finite number of coupon tests. The Bayesian technique is used to update the failure stress distribution based on results of the element tests. Structural design following the FAA regulations is considered and the tradeoffs between the number of tests, weight and probability of failure in certification and in service are explored. We find that these are not very sensitive to the number of tests.
\end{abstract}

\section{Nomenclature}

$A=$ load carrying area of a small part of the overall structure

$b_{t}=$ bound of error in the design thickness, $e_{t}$

$b_{w}=$ bound of error in the design width, $e_{w}$

$e_{e f}=$ error associated with failure criterion used while predicting failure in the structural element tests

$e_{f}=$ error in predicting failure of the entire structure in certification or proof test

$e_{p}=$ error in load calculation

$e_{\sigma}=$ error in stress calculation

$e_{t}=$ error in the design thickness due to construction errors

$e_{w}=$ error in the design width due to construction errors

$E() \quad=$ expected value (i.e., mean value)

$P_{\text {calc }}=$ calculated design load

$P_{d}=$ true design load based on the FAA specifications (e.g., gust load specification)

$P_{f}=$ probability of failure

$P F C T=$ probability of failing certification test

$\sigma_{c a}=$ allowable stress (B-basis) from coupon testing

$\sigma_{e a}=$ allowable stress (B-basis) from element testing

$\sigma_{a}=$ allowable stress (B-basis) of the entire structure

$\sigma_{c f}=$ failure stress from coupon testing

$\sigma_{e f}=$ failure stress of the structural element

$\left(\sigma_{e f}\right)^{\text {test }}=$ element failure stress measured in tests

$\left(\sigma_{e f}\right)_{c a l c}=$ calculated (or predicted) element failure stress

$\left(\sigma_{e f}\right)^{\text {upd }}=$ updated value of the calculated (or predicted) element failure stress

$\sigma_{f}=$ failure stress of the overall structure

$\sigma=$ stress in a small part of the overall structure

$k_{d}=$ knockdown factor at coupon level due to use of conservative (B-basis) material properties

${ }_{1}^{1}$ Assistant Professor, Mechanical Engineering, AIAA Member.

${ }^{2}$ Distinguished Professor, Mechanical and Aerospace Engineering, AIAA Fellow.

${ }_{3}^{3}$ Associate Professor, Mechanical and Aerospace Engineering, AIAA Member.

${ }^{4}$ MS Student, Mechanical and Aerospace Engineering. 
$k_{f}=$ additional knockdown factor at the structural level (taken 0.9 here)

$S_{F}=$ the FAA load safety factor of 1.5

$t=$ thickness of a small part of the overall structure

$v_{t}=$ effect of variability on the built thickness

$v_{w}=$ effect of variability on the built width

$w$ = width of a small part of the overall structure

Subscripts

built-av = average built value that differs from the design value due to errors in construction

built-var = actual built value that differs from the average built value due to variabilities in construction

calc = calculated (or predicted) value that differs from the design value due to errors in design

design $=$ design value

true $=$ true value (error free value)

\section{Introduction}

$\mathrm{T}$

HE safety of aircraft structure can be achieved by designing the structure against uncertainty and by taking steps to reduce the uncertainty. Safety factors and knockdown factors are examples of measures used to compensate for uncertainty during the design process. Uncertainty reduction measures (URMs), on the other hand, may be employed during the design process or later on throughout the operational lifetime. Examples of URMs for aircraft structural systems include structural testing, quality control, inspection, health monitoring, maintenance, and improved structural analysis and failure modeling.

In reliability-based design optimization, all uncertainties that are available at the design stage are considered in calculating the reliability of the structure. However, the actual aircraft is much safer, because after the design it is customary to engage in vigorous uncertainty reduction activities using various URMs. It would be therefore beneficial to include the effects of these planned URMs in the design process. It may be even advantageous to design the URMs together with the structure; trading off the cost of more weight against additional tests or more refined analytical simulations. It is challenging, though, to model the effect of future URMs in the design process.

In probabilistic design, the contribution of each URM can be represented by a distribution. In the case of predesign tests, since the tests have already been performed, the parameters (e.g., mean and variance) of the distribution are known and can be used to predict the distribution of failure stress. In the case of future tests, however, the parameters of the distribution are unknown, and the same is true for other future URMs. In this case, these parameters can be considered as random variables. Then, a future URM can be modeled as a distribution of distributions.

The objective of the present paper is (1) to explore modeling the effect of future tests on uncertainty in structural failure predictions and (2) to study their effects on structural design. Since the distribution type of failure stress is unknown a priori, we use one of the most general distribution types, Johnson distribution, which can be represented by four quartiles. As mentioned above, since the distribution parameters of future tests are random, the four quartiles of the failure stress distributions are modeled as normal distributions. Then, the distribution of these four quartiles will depend on the number of tests; more tests will reduce the variance of quartile distributions. Then, the critical information for tradeoff analysis will be how much uncertainty can be reduced by a given number of future tests.

In the paper we investigate in particular the effect of the number of coupon and structural element tests on the final distribution of the failure stress. It is assumed that the mean value of the failure stress (mean over a large number of aircraft) is obtained from a failure criterion (e.g., Tsai-Wu theory [1]) using the results of coupon tests. The initial uncertainty in this mean failure stress reflects the confidence of the analytical model in this prediction. The Bayesian technique is then used to update the mean failure stress distribution from the results of the element tests. In addition, there is the variability of the failure stress from one aircraft to another or from one structural component to another. We assume that this variability is the same as the variability in coupon tests. However, this variability is not fully known because of the finite number of coupon tests. 
Finally, we consider structural design following the FAA regulations (using B-basis allowables). We show tradeoffs between the number of tests and the weight of the structure for a given probability of failure. These could provide tradeoffs between additional tests and heavier weight depending on the cost of testing and the cost of carrying the additional weight.

The paper is organized as follows. Section II discusses the safety measures taken during aircraft structural design. Section III presents a simple uncertainty classification that distinguishes uncertainties that affect an entire fleet (errors) from the uncertainties that vary from one aircraft to another in the same fleet (variability). Section IV discussed modeling of errors and variability throughout the design and testing of an aircraft. Section V discusses probability of failure estimation via Monte Carlo simulations. Finally, the results and the concluding remarks are given in the last two sections of the paper, respectively.

\section{Safety Measures}

The safety of aircraft structures is achieved by designing these structures to operate well in the presence of uncertainties and taking steps to reduce the uncertainties. The following gives brief description of these safety measures.

\section{A. Safety measures for designing structures under uncertainties}

Load Safety Factor: In transport aircraft design, FAA regulations state the use of a load safety factor of 1.5 (FAR-25.303 [2]). That is, aircraft structures are designed to withstand 1.5 times the limit load without failure.

Conservative Material Properties: In order to account for uncertainty in material properties, FAA regulations state the use of conservative material properties (FAR-25.613 [3]). The conservative material properties are characterized as A-basis and/or B-basis material property values. Detailed information on these values is provided in Volume 1, Chapter 8 of the Composite Materials Handbook [4]. In this paper, we use B-basis values. The B-basis value is determined by calculating the value of a material property exceeded by $90 \%$ of the population with $95 \%$ confidence. The basis values are determined by testing a number of coupons selected randomly from a material batch. In this paper, the nominal value of the number of coupon tests is taken 50. In the Results section, the effect of the number of coupon tests will be explored.

Other measures such as redundancy are not discussed in this paper.

\section{B. Safety measures for reducing uncertainties}

Improvements in accuracy of structural analysis and failure prediction of aircraft structures reduce errors and enhance the level of safety of the structures. These improvements may be due to better modeling techniques developed by researchers, more detailed finite element models made possible by faster computers, or more accurate failure theories. Similarly, the variability in material properties can be reduced through quality control and improved manufacturing processes. Variability reduction in damage and ageing effects is accomplished through inspections and structural health monitoring. The reader is referred to the papers by Acar et al. [5] for effects of error reduction, Qu et al. [6] for effects of variability reduction, and Acar et al. [7] for effects of reduction of both error and variability.

In this paper, we focus on error reduction through aircraft structural tests, while the other uncertainty reduction measures are left out for future studies. Structural tests are conducted in a building block procedure (Volume I, Chapter 2 of Ref. [4]). First, individual coupons are tested to estimate the mean and variability in failure stress. The mean structural failure is estimated based on

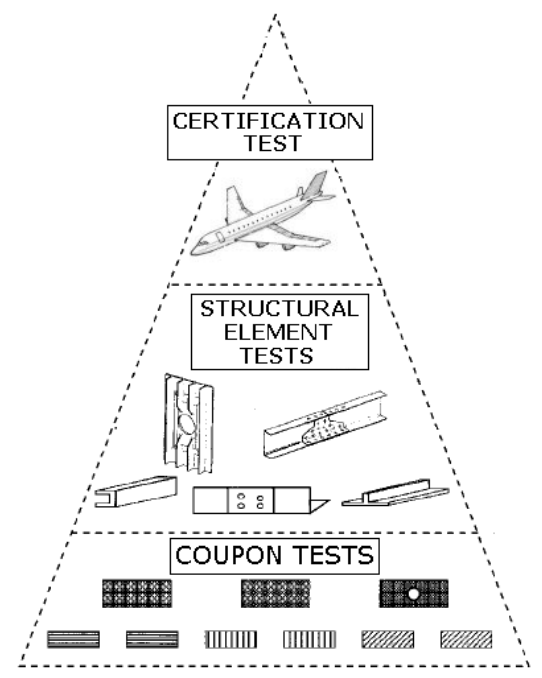

Figure 1. Simplified three-level tests 
failure criteria (such as Tsai-Wu) and this estimate is further improved using element tests. Then a sub-assembly is tested, followed by a full-scale test of the entire structure. In this paper, we use the simplified three-level test procedure depicted in Figure 1. The coupon tests, structural element tests and the final certification test are included.

The first level is the coupon tests, where coupons (i.e., material samples) are tested to estimate failure stress. The FAA regulation FAR 25-613 requires aircraft companies to perform "enough" tests to establish design values of material strength properties (A-basis or B-basis value). As the number of coupon tests increases, the errors in the assessment of the material properties are reduced. However, since testing is costly, the number of coupon tests is limited to about 100 to 300 for A-basis calculation and 30+ (i.e., more than 30) for B-basis value calculation. In this paper, the nominal value of the number of coupon tests is taken 50 .

At the second level of testing, structural elements are tested. The main target of element tests is to reduce errors related to failure theories (e.g., Tsai-Wu) used in assessing the failure load of the structural elements. In this paper, the nominal value of the number of structural element tests is taken as 3 .

At the uppermost level, certification (or proof) testing of the overall structure is conducted (FAR 25-307 [8]). This final certification or proof testing is intended to reduce the chance of failure in flight due to errors in the structural analysis of the overall structure (e.g., errors in finite element analysis, errors in failure mode prediction). While failure in flight often has fatal consequences, certification failure often has serious financial implications. So we measure the success of the URMs in terms of their effect on probability of failure in flight and in terms of their effect on probability of certification failure.

\section{Structural Uncertainties}

A good analysis of different sources of uncertainty in engineering simulations is provided by Oberkampf et al. $[9,10]$. To simplify the analysis, we use a classification that distinguishes between errors (uncertainties that apply equally to the entire fleet of an aircraft model) and variability (uncertainties that vary for the individual aircraft) as we used in our earlier studies [11-12]. The distinction, presented in Table 1, is important because safety measures usually target either errors or variability. While variabilities are random uncertainties that can be readily modeled probabilistically, errors are fixed for a given aircraft model (e.g., Boeing 737-400) but they are largely unknown. Since errors are epistemic, they are often modeled using fuzzy numbers or possibility analysis. We model errors probabilistically by using uniform distributions to maximize the entropy.

Table 1. Uncertainty Classification

\begin{tabular}{cccc}
\hline \hline $\begin{array}{c}\text { Type of } \\
\text { Uncertainty }\end{array}$ & Spread & Cause & Remedies \\
\hline $\begin{array}{c}\text { Error } \\
\text { (mostly } \\
\text { epistemic) }\end{array}$ & $\begin{array}{c}\text { Departure of the average } \\
\text { fleet of an aircraft model } \\
\text { (e.g. Boeing 737-400) from } \\
\text { an ideal }\end{array}$ & $\begin{array}{c}\text { Errors in predicting } \\
\text { structural failure, } \\
\text { construction errors, } \\
\text { deliberate changes }\end{array}$ & $\begin{array}{c}\text { Testing and simulation to } \\
\text { improve the mathematical } \\
\text { model and the solution }\end{array}$ \\
\hline $\begin{array}{c}\text { Variability } \\
\text { (aleatory) }\end{array}$ & $\begin{array}{c}\text { Departure of an individual } \\
\text { aircraft from fleet level } \\
\text { average }\end{array}$ & $\begin{array}{c}\text { Variability in tooling, } \\
\text { manufacturing process, } \\
\text { and flying environment }\end{array}$ & $\begin{array}{c}\text { Improvement of tooling } \\
\text { and construction. Quality } \\
\text { control }\end{array}$ \\
\hline \hline
\end{tabular}

Errors are uncertain at the time of the design but they will not vary for a single structural component on a particular aircraft, while the variabilities vary for individual structural components. To model errors, we assume that we have a large number of nominally identical aircraft being designed (e.g., by Airbus, Boeing, Embraer, Bombardier, etc.), with the errors being fixed for each aircraft.

\section{Modeling Errors and Variability}

\section{A. Errors in estimating material strength properties from coupon testing}

Coupon tests are conducted to obtain the statistical characterization of material strength properties, such as failure stress, and their corresponding design values (A-basis or B-basis). With a finite number $n_{c}$ of coupon tests, 
the statistical characterization involves errors. Therefore, the calculated values of the mean and the standard deviation of the failure stress will be uncertain. We assume that the failure stress follows normal distribution, so the calculated mean also follows normal distribution. In addition, when $n_{c}$ is larger than 25 , the distribution of the calculated standard deviation tends to be normal. Then, the calculated failure stress can be expressed as

$$
\left(\sigma_{c f}\right)_{\text {calc }}=\text { Normal }\left[\left(\bar{\sigma}_{c f}\right)_{\text {calc }} ; \operatorname{Std}\left(\sigma_{c f}\right)_{\text {calc }}\right]
$$

where calculated mean and the calculated apparent standard deviation can be expressed as

$$
\left.\begin{array}{c}
\left(\bar{\sigma}_{c f}\right)_{\text {calc }}=\operatorname{Normal}\left(\bar{\sigma}_{f} ; \frac{\operatorname{Std}\left(\sigma_{f}\right)}{\sqrt{n_{c}}}\right) \\
\operatorname{Std}\left(\sigma_{c f}\right)_{\text {calc }}=\operatorname{Normal}\left(\operatorname{Std}\left(\sigma_{f}\right) \sqrt{\frac{1+\sqrt{\frac{n_{c}-3}{n_{c}-1}}}{2}} ; \operatorname{Std}\left(\sigma_{f}\right) \sqrt{\frac{1-\sqrt{\frac{n_{c}-3}{n_{c}-1}}}{2}}\right.
\end{array}\right)
$$

where $\bar{\sigma}_{f}$ and $\operatorname{Std}\left(\sigma_{f}\right)$ are, respectively, the true values of the mean and standard deviation of failure stress. Note that Eqs. (1)-(3) describe a random variable coming from a distribution (normal) whose parameters are also random. In this paper, this will be referred to as a distribution of distributions.

The allowable stress at the coupon level, $\sigma_{c a}$, is computed from the failure stress calculated at the coupon level, $\left(\bar{\sigma}_{c f}\right)_{\text {calc }}$, by using a knockdown factor, $k_{d}$, as

$$
\sigma_{c a}=k_{d}\left(\bar{\sigma}_{c f}\right)_{c a l c}
$$

The knockdown factor $k_{d}$ is specified by the FAA regulations (FAR). For instance, for the B-basis value of the failure stress, $90 \%$ of the failure stresses (measured in coupon tests) must exceed the allowable stress with $95 \%$ confidence. The requirement of $90 \%$ probability and $95 \%$ confidence is responsible for the knockdown factor $k_{d}$ in Eq. (4). For normal distribution, the knockdown factor depends on the number coupon tests and the c.o.v. of the failure stress as

$$
k_{d}=1-k_{B}\left(c_{c f}\right)_{c a l c}
$$

where $\left(c_{c f}\right)_{\text {calc }}$ is the c.o.v. of failure stress calculated from coupon tests. The tolerance coefficient $k_{B}$ is a function of the number of coupon tests $n_{c}$ as given in Ref. [4] (Volume 1, Chapter 8, page 84) as

$$
k_{B} \approx 1.282+\exp \left(0.958-0.520 \ln \left(n_{c}\right)+\frac{3.19}{n_{c}}\right)
$$

\section{B. Errors in structural element strength predictions}

The second level in the testing sequence is structural element testing, where structural elements are tested to validate the accuracy of the failure criterion used (e.g., Tsai-Wu). Here, we assume that structural element tests are conducted for a specified combination of loads corresponding to critical loading. For this load combination, the failure surface can be boiled down to a single failure stress $\bar{\sigma}_{e f}$ where the subscript ' $e$ ' stands for structural element tests. 
If the failure theory used to predict the failure was perfect, and we performed infinite number of coupon tests, then we could predict the true mean element failure stress at the structural element tests. The actual value would vary only due to material variability. However, neither the failure theory is perfect nor infinite tests are performed, so the calculated value of the mean failure stress at the element level is

$$
\left(\bar{\sigma}_{e f}\right)_{c a l c}=\left(1-e_{e f}\right)\left(\bar{\sigma}_{c f}\right)_{c a l c}
$$

where $e_{e f}$ is the error in the failure theory. Note that the sign in front of the error term is negative, since we consistently formulate the error expressions such that a positive error implies a conservative decision. The initial distribution of $\left(\bar{\sigma}_{e f}\right)_{\text {calc }}$ is obtained by estimate of the error $e_{e f}$ and using the results of coupon tests $\left(\bar{\sigma}_{c f}\right)_{\text {calc }}$. The information from element tests is used by performing Bayesian procedure to update the failure stress distribution (see An et al. [13] for details). In practice, simpler procedures are often used, such as selecting the lowest failure stress from element tests. Therefore, our assumption will tend to overestimate the beneficial effect of element tests.

The allowable stress based on the element test is calculated from

$$
\sigma_{e a}=k_{d}\left(\bar{\sigma}_{e f}\right)_{\text {calc }}^{\text {updated }}
$$

where the $\left(\bar{\sigma}_{e f}\right)_{\text {calc }}^{\text {updated }}$ is the value of the mean failure stress corresponding to the maximum PDF.

\section{Redesign based on element tests:}

Besides updating the failure stress, element tests have an important role of leading to design changes if the design is unsafe or overly conservative. That is, if very large or very small failure stress values are obtained from the element tests, the company may want to increase or reduce the thicknesses of the elements. We did not find published data on redesign practices, and so we devised a common sense approach reflected in Table 2. We assumed that if the B-basis value obtained after element tests, $\sigma_{e a}$, is more than $5 \%$ higher than the B-basis value obtained from coupon tests, $\sigma_{c a}$, then the element thickness is reduced by $\sigma_{c a} / \sigma_{e a}$ ratio. If the B-basis value obtained after element tests is more than $2 \%$ lower than the B-basis value obtained from coupon tests, the element thickness is increased by $\sigma_{c a} / \sigma_{e a}$ amount. This lower tolerance reflects the need for safety. Otherwise, no redesign was performed. The thickness of the element is obtained through

$$
\begin{gathered}
t_{\text {elem }}=\left(1-e_{e f}\right) \frac{S_{F} P_{d}}{w} \frac{1}{\sigma_{e a}} \text { (no redesign) } \\
t_{\text {elem }}=\left(1-e_{e f}\right) \frac{S_{F} P_{d}}{w \sigma_{e a 2}} \frac{1.01}{C . F .}=\left(1-e_{e f}\right) \frac{S_{F} P_{d}}{w} \frac{1.01 \sigma_{e a}}{\sigma_{c a} \sigma_{e a 2}} \text { (redesign) }
\end{gathered}
$$

where $S_{F}$ is the load safety factor, $P_{d}$ is the design load for testing the elements. Since redesign requires new elements to be built and tested, it is costly. Therefore, we do not have a redesign over redesigned elements. In order to protect against uncertainties in the test of the redesigned element we have an additional $1 \%$ reduction in the calculated allowable value (see the term 1.01 in Eq. (8)).

Table 2. Simulation of element tests

1. Generate random numbers for the quartiles of the mean failure stress

2. Calculate the B-basis value using the quartiles

3. Check to see if redesign is needed

4. If redesign is needed

a. Generate new random numbers for the quartiles

b. Calculate the new B-basis value using the new quartiles

5. Compute the design thickness of the element

6. Compute probabilities of failure in certification tests and under service loads 


\section{Errors in structural strength predictions}

Due to the complexity of the overall structural system, there will be errors in failure prediction of the overall structure that we denote as $e_{f}$. Therefore, the calculated value of the failure stress of the overall structure, $\left(\vec{\sigma}_{f}\right)_{\text {calc }}$, can be expressed as

$$
\left(\vec{\sigma}_{f}\right)_{\text {calc }}=\left(1-e_{f}\right)\left(\bar{\sigma}_{e f}\right)_{\text {calc }}
$$

The allowable stress at the structural design level, $\sigma_{a}$, can be related to the allowable stress computed at the element level, $\sigma_{e a}$, through the following relation

$$
\sigma_{a}=k_{f}\left(1-e_{f}\right) \sigma_{e a}
$$

where $k_{f}$ is an additional knockdown factor used at the structural level as an extra precaution. Here $k_{f}$ is taken 0.9.

\section{Errors in design}

We consider static point stress design for simplicity. Other types of failures such as fatigue, corrosion or crack instability are not taken into account. Before starting the structural design, aerodynamic analysis needs to be performed to determine the loads acting on the aircraft. However, the calculated design load value, $P_{\text {calc }}$, differs from the actual loading $P_{d}$ under conditions corresponding to FAA design specifications (e.g., gust-strength specifications). Since each company has different design practices, the error in load calculation, $e_{p}$, is different from one company to another. The calculated design load $P_{\text {calc }}$ is expressed in terms of the true design load $P_{d}$ as

$$
P_{\text {calc }}=\left(1+e_{P}\right) P_{d}
$$

Notice here that the sign in front of the load error term is positive while the sign in front of the failure stress error terms were negative. The reason for this choice, as we noted earlier, is that we consistently formulate the error expressions such that a positive error implies a conservative decision.

Besides the error in load calculation, an aircraft company may also make errors in stress calculation. We consider a small region in a structural part, characterized by a thickness $t$ and width $w$, that resists the load in that region. The value of the stress in a structural part calculated by the stress analysis team, $\sigma_{\text {calc }}$, can be expressed in terms of the load values calculated by the load team $P_{\text {calc }}$, the design width $w_{\text {design }}$, and the thickness $t$ of the structural part by introducing the term $e_{\sigma}$ representing error in the stress analysis

$$
\sigma_{\text {calc }}=\left(1+e_{\sigma}\right) \frac{P_{\text {calc }}}{w_{\text {design }} t}
$$

In this paper, we assume that the aircraft companies have the capability of predicting the stresses very accurately so that the effect of $e_{\sigma}$ is negligible and is taken as zero. The calculated stress value is then used by a structural designer to calculate the design thickness $t_{\text {design }}$ required to carry the calculated design load times the safety factor $S_{F}$. That is,

$$
t_{\text {design }}=\frac{S_{F} P_{\text {calc }}}{w_{\text {design }} \sigma_{a}}=\frac{\left(1+e_{P}\right)}{\left(1-e_{f}\right)} \frac{S_{F} P_{d}}{w_{\text {design }} k_{f} \sigma_{e a}}
$$

From Eq. (13), we can express the design value of the load carrying area as

$$
A_{\text {design }}=t_{\text {design }} w_{\text {design }}=\frac{\left(1+e_{P}\right)}{\left(1-e_{f}\right)} \frac{S_{F} P_{d}}{k_{f} \sigma_{e a}}
$$




\section{E. Errors in construction}

In addition to the above errors, there will also be construction errors in the geometric parameters. These construction errors represent the difference between the values of these parameters in an average airplane (fleetaverage) built by an aircraft company and the design values of these parameters. The error in width, $e_{w}$, represents the deviation of the design width of the structural part, $w_{\text {design }}$, from the average value of the width of the structural part built by the company, $w_{\text {built }-a v}$. Thus,

$$
w_{\text {built }-a v}=\left(1+e_{w}\right) w_{\text {design }}
$$

Similarly, the built thickness value will differ from its design value such that

$$
t_{\text {built }-a v}=\left(1+e_{t}\right) t_{\text {design }}
$$

Then, the built load carrying area $A_{\text {builtav }}$ can be expressed using the first equality of Eq. (14) as

$$
A_{\text {built-av }}=\left(1+e_{t}\right)\left(1+e_{w}\right) A_{\text {design }}
$$

Table 3 presents nominal values for the errors assumed here. In the results section of the paper we will vary these error bounds and investigate the effects of these changes on the probability of failure.

Table 3. Distribution of error terms and their bounds

\begin{tabular}{cccc}
\hline \hline Error factors & Distribution Type & Mean & Bounds \\
\hline Error in load calculation, $e_{P}$ & Uniform & 0.0 & $\pm 10 \%$ \\
Error in width, $e_{w}$ & Uniform & 0.0 & $\pm 1 \%$ \\
Error in thickness, $e_{t}$ & Uniform & 0.0 & $\pm 3 \%$ \\
Error in failure prediction, $e_{f}$ & Uniform & 0.0 & $\pm 10 \%$ \\
Error in failure prediction, $e_{e f}$ & Uniform & 0.0 & $\pm 10 \%$ \\
\hline \hline
\end{tabular}

The errors here are modeled by uniform distributions, following the principle of maximum entropy. For instance, the error in the built thickness of a structural part $\left(e_{t}\right)$ is defined in terms of the error bound $\left(b_{t}\right)_{\text {built }}$ using

$$
e_{t}=U\left[0,\left(b_{t}\right)_{b u i l t-a v}\right]
$$

Here ' $U$ ' indicates that the distribution is uniform, ' 0 (zero)' is the average value of $e_{t}$, and the error bound is $\left(b_{t}\right)_{\text {built-av }}=0.03$. Hence, the lower bound for the thickness value is the average value minus $3 \%$ of the average and the upper bound for the thickness value is the average value plus $3 \%$ of the average. Commonly available random number generators provide random numbers uniformly distributed between 0 and 1 . Then, the error in the built thickness can be calculated using such random numbers $r$ as

$$
e_{t}=(2 r-1)\left(b_{t}\right)_{b u i l t-a v}
$$

\section{F. Total error, $\boldsymbol{e}_{\text {total }}$}

The expression for the built load carrying area, $A_{\text {built-av }}$, of a structural part can be reformulated by combining Eqs. (14) and (17) as

$$
A_{b u i l t-a v}=\left(1+e_{\text {total }}\right) \frac{S_{F} P_{d}}{k_{f} \sigma_{e a}}
$$

where 


$$
e_{\text {total }}=\frac{\left(1+e_{P}\right)\left(1+e_{t}\right)\left(1+e_{w}\right)}{\left(1-e_{f}\right)}-1
$$

Here $e_{\text {total }}$ represents the cumulative effect of the individual errors on the load carrying capacity of the structural part.

\section{G. Variability}

In the previous sections, we analyzed the different types of errors made in the design and construction stages, representing the differences between the fleet average values of geometry, material and loading parameters and their corresponding design values. For a given design, these parameters vary from one aircraft to another in the fleet due to variability in tooling, construction, flying environment, etc. For instance, the actual value of the thickness of a structural part, $t_{\text {built-var }}$, is defined in terms of its fleet average built value, $t_{\text {built-av }}$, by

$$
t_{\text {built -var }}=\left(1+v_{t}\right) t_{\text {built-av }}
$$

We assume that $v_{t}$ has a uniform distribution with 3\% bounds (see Table 4). Then, the actual load carrying area $A_{\text {built-var }}$ can be defined as

$$
A_{\text {built }-\mathrm{var}}=t_{\text {built }-\mathrm{var}} w_{\text {built }-\mathrm{var}}=\left(1+v_{t}\right)\left(1+v_{w}\right) A_{\text {built }-a v}
$$

where $v_{w}$ represents effect of the variability on the fleet average built width.

Table 4 presents the assumed distributions for variabilities. Note that the thickness error in Table 3 is uniformly distributed with bounds of $\pm 3 \%$. Thus the difference between all thicknesses over the fleets of all companies is up to $\pm 6 \%$. However, the combined effect of the uniformly distributed error and variability is not uniformly distributed.

Table 4. Distribution of random variables having variability

\begin{tabular}{cccc}
\hline \hline Variables & Distribution type & Mean & Scatter \\
\hline Actual service load, $P_{a c t}$ & Lognormal & $P_{d}=2 / 3$ & $10 \%$ c.o.v. \\
Actual built width, $w_{\text {built } \text {-var }}$ & Uniform & $w_{\text {built-av }}$ & $1 \%$ bounds \\
Actual built thickness, $t_{\text {built-var }}$ & Uniform & $t_{\text {built-av }}$ & $3 \%$ bounds \\
Failure stress, $\sigma_{f}$ & Normal & 1.0 & $8 \%$ c.o.v. \\
$v_{w}$ & Uniform & 0 & $1 \%$ bounds \\
$v_{t}$ & Uniform & 0 & $3 \%$ bounds \\
\hline \hline
\end{tabular}

c.o.v. $=$ coefficient of variation

\section{H. Certification test}

After a structural part has been built with random errors in stress, load, width, allowable stress and thickness, it may fail in certification testing part of the airplane. Recall that the structural part will not be manufactured with complete fidelity to the design due to variability in the geometric properties. That is, the actual values of these parameters $w_{\text {built-var }}$ and $t_{\text {built-var }}$ will be different from their fleet-average values $w_{\text {builtav }}$ and $t_{\text {built-av }}$ due to variability. The structural part is then loaded with the design axial force of $S_{F}$ times $P_{\text {calc }}$, and if the stress exceeds the failure stress of the structure $\sigma_{f}$, then the structure fails and the design is rejected; otherwise it is certified for use. That is, the structural part is certified if the following inequality is satisfied

$$
\sigma-\sigma_{f}=\frac{S_{F} P_{\text {calc }}}{w_{\text {built }- \text { var }} t_{\text {built }- \text { var }}}-\sigma_{f} \leq 0
$$




\section{Probability of Failure Calculation by Separable MCS}

To calculate the probability of failure, we first incorporate the statistical distributions of errors and variability in a Monte Carlo simulation. Errors are uncertain at the time of design, but do not change for individual realizations (in actual service) of a particular design. On the other hand, all individual realizations of a particular design are different from each other due to variability. The simulation of error and variability can be easily implemented through a twolevel Monte Carlo simulation [11]. At the upper level different aircraft companies can be simulated by assigning random errors to each, and at the lower level we simulated variability in dimensions, material properties, and loads related to manufacturing variability and variability in service conditions can be simulated.

The effect of element tests on failure stress distribution is modeled using Bayesian updating. If the Bayesian updating is used directly within an MCS loop for design thickness determination, the computational cost will be very high. In this paper, instead, the Bayesian updating is performed aside in a separate MCS, before starting with the MCS loop for design thickness determination (the details of thickness calculation is provided in Table 5). The procedure followed for Bayesian updating can be described briefly as follows. First, the four quartiles of the mean failure stress are modeled as normal distributions. Then, these quartiles are used to fit a Johnson distribution to the mean failure stress. That is, the mean failure stress is represented as a Johnson distribution, whose parameters are themselves distributions that depend on the quality of the tests. Finally, Bayesian updating is used to update the mean failure stress distribution. Details of this procedure are provided in Appendix.

Table 5. Detailed simulation of element tests

1. Generate random numbers for the quartiles of the mean failure stress (see Appendix)

2. Calculate the B-basis value using the quartiles, $\sigma_{e a}$

a. Compute the bounds for mean failure stress $l b=0.9\left(1-e_{e f}\right)$ and $u b=1.1\left(1-e_{e f}\right)$

b. Compute the PDF of the mean failure stress having Johnson distribution within the bounds, and select the mean failure stress value with the highest PDF

c. Compute B-basis value, $\sigma_{e a}=\left[1-k_{B}\left(c_{c f}\right)_{\text {calc }}\right]\left(\bar{\sigma}_{e f}\right)_{\text {calc }}^{\text {updated }}$

3. If redesign is selected as an option, check to see if redesign is needed

a. Compute a correction factor for the B-basis value, C.F. $=\frac{\sigma_{e a}}{\sigma_{c a}}$. Limit the value of the correction factor to $[0.9,1.1]$.

b. If $C . F .<0.98=>$ redesign is needed, we will increase the thickness by C.F.

If $0.98<C . F .<1.05=>$ no redesign is needed

If $C . F .>1.05=>$ redesign is needed, we will decrease the thickness by C.F.

4. If redesign is needed

a. Generate new random numbers for the quartiles

b. Calculate the new B-basis value using the new quartiles, $\sigma_{e a 2}$ (see Step 2).

5. Compute the design thickness of the element

a. If no redesign, $t_{\text {elem }}=\left(1-e_{e f}\right) \frac{S_{F} P_{d}}{w} \frac{1}{\sigma_{e a}}$

b. If redesign, $t_{e l e m}=\left(1-e_{e f}\right) \frac{S_{F} P_{d}}{w \sigma_{e a 2}} \frac{1.01}{C . F .}=\left(1-e_{e f}\right) \frac{S_{F} P_{d}}{w} \frac{1.01 \sigma_{e a}}{\sigma_{c a} \sigma_{e a 2}}$

The prediction of probability of failure via the conventional Monte Carlo procedure requires trillions of simulations for the level of $10^{-7}$ failure probability. In order to address the computational burden, separable Monte Carlo procedure can be used [12]. The reader is referred to Smarslok and Haftka [14] for more information on the separable Monte Carlo procedure. This procedure applies when the failure condition can be expressed as $g_{1}\left(x_{1}\right)>g_{2}\left(x_{2}\right)$, where $x_{1}$ and $x_{2}$ are two disjoint sets of random variables. To take advantage of this procedure, we need to formulate the failure condition in a separable form, so that $g_{1}$ will depend only on variabilities and $g_{2}$ only on errors. The common formulation of the structural failure condition is in the form of a stress exceeding the material limit. This form, however, does not satisfy the separability requirement. For example, the stress depends on 
variability in material properties as well as design area, which reflects errors in the analysis process. To bring the failure condition to the right form, we instead formulate it as the required cross sectional area $A_{\text {req }}^{\prime}$ being larger than the built area $A_{\text {built-av }}$, as given in Eq (25).

$$
A_{\text {built }-a v}<\frac{A_{\text {req }}}{\left(1+v_{t}\right)\left(1+v_{w}\right)} \equiv A_{r e q}^{\prime}
$$

where $A_{\text {req }}$ is the cross-sectional area required to carry the actual loading conditions for a particular copy of an aircraft model, and $A_{\text {req }}^{\prime}$ is what the built area (fleet-average) needs to be in order for the particular copy to have the required area after allowing for variability in width and thickness.

$$
A_{\text {req }}=P / \sigma_{f}
$$

The required area depends only on variability, while the built area depends only on errors. When certification testing is taken into account, the built area, $A_{b u i l t-a v}$, is replaced by the certified area, $A_{c e r t}$, which is the same as the built area for companies that pass certification. However, companies that fail are not included. That is, the failure condition is written as

$$
\begin{aligned}
\text { failure without certification tests: } & A_{\text {built }-a v}-A_{\text {req }}^{\prime}<0 \\
\text { failure with certification tests: } & A_{\text {cert }}-A_{r e q}^{\prime}<0
\end{aligned}
$$

The separable Monte Carlo simulation procedure is summarized in Figure 2.

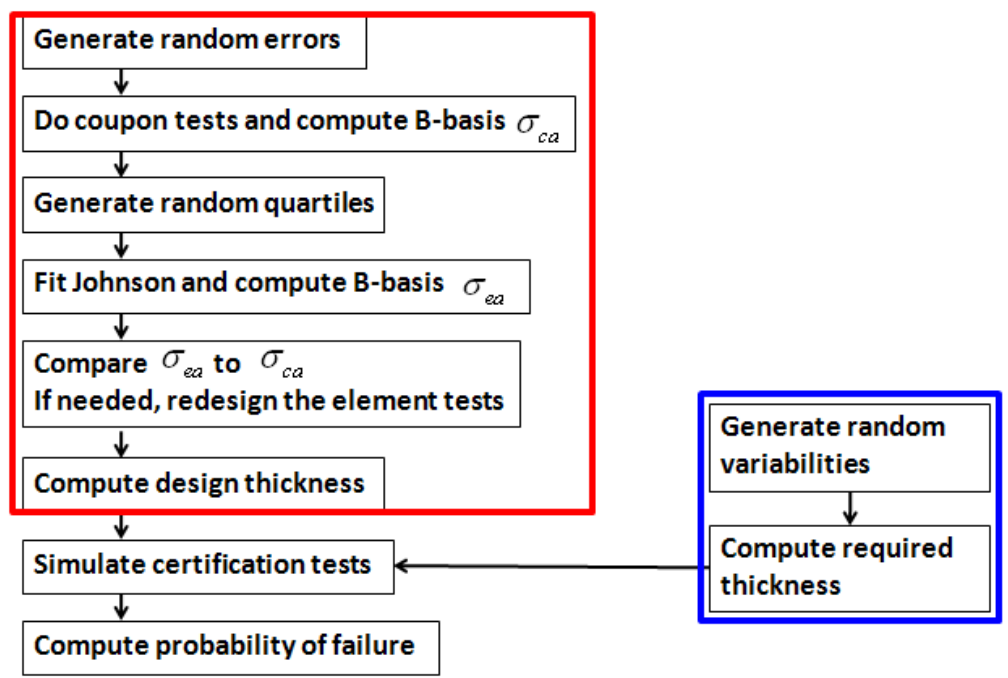

Figure 2. Flowchart for separable Monte Carlo simulations

\section{Results}

In this section, the effects of the number of coupon tests, the number of element tests, redesign of element tests and certification test are reported. As noted earlier, the nominal values of the number of coupon tests and the number of element tests are taken as 50 and 3, respectively. The redesign of element tests and the certification test are included in the analysis except for the cases that investigated the effect of redesign of element tests and the certification test. 


\section{A. Effect of the number of coupon tests}

The effects of increasing the number of coupon tests on the thickness and probability of failure are presented in Table 6. The thickness values provided in Table 6 are based on the load and material property values assumed in Table 5. As the number of coupon tests increases, both the mean thickness is reduced (since B-basis value is increased), the coefficient of variation of the thickness is reduced (since the coefficient of variation of the B-basis value is reduced). These two reductions have opposing effect on the probability of failure, and the probability of failing in certification. However, the net effect is that both probabilities of failure increase. This reflects the fact that the knockdown factor used by the FAA to compensate for small number of coupon tests (Eq. 6) is conservative, so performing more tests, actually makes the aircraft less safe!

Table 6. Effects of the number of coupon tests. Number of element tests, $n_{c}$, is 3. Redesign of element tests and certification test are included in the analysis.

\begin{tabular}{|c|c|c|c|c|}
\hline $\boldsymbol{n}_{\boldsymbol{c}}$ & $\boldsymbol{t}_{\text {mean }}$ & $\boldsymbol{t}_{\text {cov }}$ & $\boldsymbol{P}_{\boldsymbol{f}}$ & $\boldsymbol{P F C T}^{*}$ \\
\hline 30 & 1.268 & 0.116 & $1.02 \times 10^{-4}$ & 0.0576 \\
\hline 50 & 1.253 & 0.114 & $1.27 \times 10^{-4}$ & 0.0654 \\
\hline 80 & 1.245 & 0.113 & $1.44 \times 10^{-4}$ & 0.0711 \\
\hline
\end{tabular}

To provide an indication of the accuracy of the numbers in Table 6, simulations are rerun with a different seed for the random number generator. Regenerated results are provided in Table 7 . We see that the mean stress results are accurate to the fourth digit, while the probabilities are only accurate to the second digit.

Table 7. Regeneration of Table 6 results by using a different seed for the random number generator.

\begin{tabular}{|c|c|c|c|c|}
\hline $\boldsymbol{n}_{\boldsymbol{c}}$ & $\boldsymbol{t}_{\text {mean }}$ & $\boldsymbol{t}_{\text {cov }}$ & $\boldsymbol{P}_{\boldsymbol{f}}$ & $\boldsymbol{P F C T}^{*}$ \\
\hline 30 & 1.268 & 0.116 & $1.03 \times 10^{-4}$ & 0.0566 \\
\hline 50 & 1.254 & 0.114 & $1.23 \times 10^{-4}$ & 0.0664 \\
\hline 80 & 1.246 & 0.113 & $1.38 \times 10^{-4}$ & 0.0702 \\
\hline \multicolumn{6}{|c}{} \\
* PFCT: Probability of failing in certification tests
\end{tabular}

Since the mean thickness reduces as the number of coupon tests increases, the aircraft builder may decide to keep the mean thickness constant. This can be achieved by adjusting the knockdown factor $k_{f}$ in Eq. (13) so as to have same mean thickness for different number of coupon tests. First, the knockdown factor $k_{f}$ is varied by -10\%, $5 \%, 5 \%$, and $10 \%$ of its nominal value and simulations are performed. Then, response surfaces are constructed for the mean thickness $\left(t_{\text {mean }}\right)$, the probability of failure $\left(P_{f}\right)$, and the probability of failing in certification test $(P F C T)$ for each value of number of coupon tests. Finally, $P_{f}$ and $P F C T$ values corresponding to the mean thickness value of 1.253 are computed. This practice also reduces the numerical noise in simulation results. Table 8 shows that increasing the number of coupon tests from 50 to 80 leads to $13 \%$ reduction in probability of failure, whereas reducing the number of coupon tests to 30 yields $20 \%$ probability of failure inflation. We can also conclude that increasing the number of coupon tests reduces the probability of failure for the same weight, but the rate of reduction diminishes with the number of tests. Overall, it appears that increasing the number of coupon tests has only small effect on the probability of failure in service or on the probability of failing certification.

Table 8. Effects of the number of coupon tests for the same weight. Number of element tests, $n_{e}$, is 3. Redesign of element tests and certification test are included in the analysis.

\begin{tabular}{|c|c|c|c|}
\hline $\boldsymbol{n}_{\boldsymbol{c}}$ & $\boldsymbol{t}_{\text {mean }}$ & $\boldsymbol{P}_{\boldsymbol{f}}$ & $\boldsymbol{P F C T}^{*}$ \\
\hline 30_adjusted & 1.253 & $1.32 \times 10^{-4}$ & 0.0685 \\
\hline 50 & 1.253 & $1.27 \times 10^{-4}$ & 0.0654 \\
\hline 80_adjusted & 1.253 & $1.22 \times 10^{-4}$ & 0.0649 \\
\hline
\end{tabular}

* PFCT: Probability of failing in certification tests 


\section{B. Effect of the number of element tests}

The effects of increasing the number of element tests on the thickness and probability of failure are presented in Table 9. Increasing the number of element tests does not have a significant effect on the mean thickness, but we just see some fluctuations in the mean thickness values due to numerical noise. The coefficient of variation of the thickness, on the other hand, is reduced significantly because of the reduction of the error term $e_{e f}$.

Table 9. Effect of the number of element tests. Number of coupon tests, $n_{c}$, is 50. Redesign of element tests and certification test are included in the analysis.

\begin{tabular}{|c|c|c|c|c|}
\hline $\boldsymbol{n}_{\boldsymbol{e}}$ & $\boldsymbol{t}_{\text {mean }}$ & $\boldsymbol{t}_{\boldsymbol{c o v}}$ & $\boldsymbol{P}_{\boldsymbol{f}}$ & $\boldsymbol{P F C T}$ \\
\hline 0 & 1.244 & 0.119 & $1.81 \times 10^{-4}$ & 0.0880 \\
\hline 1 & 1.257 & 0.119 & $1.37 \times 10^{-4}$ & 0.0714 \\
\hline 2 & 1.254 & 0.115 & $1.29 \times 10^{-4}$ & 0.0676 \\
\hline 3 & 1.253 & 0.114 & $1.27 \times 10^{-4}$ & 0.0654 \\
\hline 4 & 1.253 & 0.112 & $1.20 \times 10^{-4}$ & 0.0637 \\
\hline 5 & 1.252 & 0.111 & $1.18 \times 10^{-4}$ & 0.0636 \\
\hline
\end{tabular}

To provide an indication of the accuracy of the numbers in Table 9, simulations are repeated with a different seed for the random number generator, as we did earlier for Table 6. Regenerated results are provided in Table 10. We see that the mean stress results are accurate to the fourth digit, while the probabilities are only accurate to the second digit.

Table 10. Regeneration of Table 9 results by using a different seed for the random number generator.

\begin{tabular}{|c|c|c|c|c|}
\hline $\boldsymbol{n}_{\boldsymbol{e}}$ & $\boldsymbol{t}_{\text {mean }}$ & $\boldsymbol{t}_{\boldsymbol{c o v}}$ & $\boldsymbol{P}_{\boldsymbol{f}}$ & $\boldsymbol{P F C T}$ \\
\hline 0 & 1.244 & 0.119 & $1.75 \times 10^{-4}$ & 0.0866 \\
\hline 1 & 1.258 & 0.119 & $1.39 \times 10^{-4}$ & 0.0713 \\
\hline 2 & 1.255 & 0.115 & $1.32 \times 10^{-4}$ & 0.0674 \\
\hline 3 & 1.253 & 0.114 & $1.29 \times 10^{-4}$ & 0.0651 \\
\hline 4 & 1.254 & 0.113 & $1.20 \times 10^{-4}$ & 0.0639 \\
\hline 5 & 1.252 & 0.112 & $1.16 \times 10^{-4}$ & 0.0625 \\
\hline
\end{tabular}

To remove the effect of the mean thickness fluctuations on the results, the mean thickness is adjusted to the nominal value of 1.253 by varying the knockdown factor $k_{f}$ in Eq. (13). Table 9 shows that increasing the number of element tests from three to five leads to a $10 \%$ reliability improvement, while reducing the number of element tests to one causes a $21 \%$ increase in probability of failure. Similar effects are observed on the probability of failing certification. It appears that three element tests (typical of present practice) is a reasonable choice.

Table 9. Effects of the number of element tests for the same weight. Number of coupon tests, $n_{c}$, is 50. Redesign of element tests and certification test are included in the analysis.

\begin{tabular}{|c|c|c|c|}
\hline $\boldsymbol{n}_{\boldsymbol{e}}$ & $\boldsymbol{t}_{\text {mean }}$ & $\boldsymbol{P}_{\boldsymbol{f}}$ & $\boldsymbol{P F C T}$ \\
\hline 0 & 1.253 & $1.55 \times 10^{-4}$ & 0.0785 \\
\hline 1 & 1.253 & $1.45 \times 10^{-4}$ & 0.0736 \\
\hline 2 & 1.253 & $1.32 \times 10^{-4}$ & 0.0691 \\
\hline 3 & 1.253 & $1.27 \times 10^{-4}$ & 0.0654 \\
\hline 4 & 1.253 & $1.20 \times 10^{-4}$ & 0.0642 \\
\hline 5 & 1.253 & $1.17 \times 10^{-4}$ & 0.0632 \\
\hline
\end{tabular}

To analyze the probability of failure and weight tradeoffs, the probability of failure can be fixed to a value and the variation of the weight with number of tests can be explored. Here, the probability of failure can be fixed to $1.27 \times 10^{-4}$, which corresponds to performing three element tests and fifty coupon tests (the nominal values). Table 10 shows that if we want to do away with element tests, then we will need to put $1 \%$ extra weight to achieve to the same probability of failure. 
Table 10. Effects of the number of element tests for the same probability of failure. Number of coupon tests, $n_{c}$, is 50. Redesign of element tests and certification test are included in the analysis.

\begin{tabular}{|c|c|c|c|c|}
\hline $\boldsymbol{n}_{\boldsymbol{e}}$ & $\boldsymbol{t}_{\text {mean }}$ & $\begin{array}{c}\text { \% increase } \text { in } \\
\text { thickness }\end{array}$ & $\boldsymbol{P}_{\boldsymbol{f}}$ & $\boldsymbol{P F C T}$ \\
\hline 0 & 1.266 & 1.0 & $1.27 \times 10^{-4}$ & 0.0653 \\
\hline 1 & 1.263 & 0.8 & $1.27 \times 10^{-4}$ & 0.0656 \\
\hline 2 & 1.256 & 0.2 & $1.27 \times 10^{-4}$ & 0.0668 \\
\hline 3 & 1.253 & --- & $1.27 \times 10^{-4}$ & 0.0654 \\
\hline 4 & 1.250 & -0.2 & $1.27 \times 10^{-4}$ & 0.0672 \\
\hline 5 & 1.249 & -0.3 & $1.27 \times 10^{-4}$ & 0.0672 \\
\hline
\end{tabular}

The probability of failing in the certification tests is high likely a big motivator for the aircraft companies, hence we also investigate how much extra weight would be needed to maintain the probability of failing in certification test if the company intends to eliminate the element tests. Table 11 shows that if a company aims to eliminate the element tests, the structural weight must be increased by $1 \%$ to achieve to the same probability of failing in certification tests.

Table 11. Effects of the number of element tests for the same probability of failing in certification test. Number of coupon tests, $n_{c}$, is 50. Redesign of element tests and certification test are included in the analysis.

\begin{tabular}{|c|c|c|c|c|}
\hline $\boldsymbol{n}_{\boldsymbol{e}}$ & $\boldsymbol{t}_{\text {mean }}$ & $\begin{array}{c}\text { \% increase in } \\
\text { thickness }\end{array}$ & $\boldsymbol{P}_{\boldsymbol{f}}$ & $\boldsymbol{P F C T}$ \\
\hline 0 & 1.266 & 1.0 & $1.28 \times 10^{-4}$ & 0.0654 \\
\hline 1 & 1.263 & 0.8 & $1.27 \times 10^{-4}$ & 0.0654 \\
\hline 2 & 1.257 & 0.3 & $1.24 \times 10^{-4}$ & 0.0654 \\
\hline 3 & 1.253 & --- & $1.27 \times 10^{-4}$ & 0.0654 \\
\hline 4 & 1.252 & -0.1 & $1.22 \times 10^{-4}$ & 0.0654 \\
\hline 5 & 1.251 & -0.2 & $1.22 \times 10^{-4}$ & 0.0654 \\
\hline
\end{tabular}

\section{Effect of the certification test}

Finally, the effect of certification test on the mean thickness and reliability are explored. Table 12 shows that if certification is not performed, then the mean thickness is reduced by a small amount while the coefficient of variation of the thickness is increased significantly. Therefore, the probability of failure is increased by almost twice. Even if the mean thickness is adjusted to its nominal value, the probability of failure is $54 \%$ larger! The overall conclusion is that the certification test is very effective of maintaining the reliability.

Table 12. Effects of certification test. Number of coupon tests $=50$. Number of element tests $=3$.

\begin{tabular}{|l|c|c|c|}
\hline & $\boldsymbol{t}_{\text {mean }}$ & $\boldsymbol{t}_{\text {cov }}$ & $\boldsymbol{P}_{\boldsymbol{f}}$ \\
\hline Certification & 1.253 & 0.114 & $1.27 \times 10^{-4}$ \\
\hline No certification & 1.244 & 0.119 & $2.31 \times 10^{-4}$ \\
\hline $\begin{array}{l}\text { No certification with } \\
\text { adjusted mean thickness }\end{array}$ & 1.253 & 0.119 & $1.94 \times 10^{-4}$ \\
\hline
\end{tabular}

\section{Concluding remarks}

The effects of aircraft structural tests on aircraft structural safety were explored. In particular, the effects of the number of coupon tests and the number of structural element tests on the final distribution of the failure stress were investigated. We simulated a structural design following the FAA regulations and explored the tradeoffs between the number of tests, weight and probability of failure. From the results obtained in this study, following conclusions can be drawn. 
- As the number of coupon tests is increased, the mean allowable stress increases so the mean thickness reduces. While the standard deviation of the thickness decreases, the probability of failure increases as does the probability of failing certification. This indicates that the FAA knockdown factor for compensating for small number of coupon tests is conservative.

- As the number of coupon tests is increased maintaining the same weight as the nominal case, the probability of failure reduces, but the rate of the reduction diminishes with the number of coupon tests. Overall, the number of coupon tests has only marginal effect on probability of failure.

- If the number of element tests is increased, the probability of failure reduces for the same weight, and the rate of this reduction decreases with the number of tests.

- If we want to dispense with element tests, then we will need to put about $1 \%$ extra structural weight to achieve to the same probability of failure.

- If certification test is not performed, the probability of failure is increased by $54 \%$, so the certification test is an effective way of maintaining the reliability.

\section{Appendix: Updating the failure stress distribution from the results of element tests}

The initial distribution of the element failure stress is obtained by using a failure criterion (e.g., Tsai-Wu theory) using the results of coupon tests. There will be two sources of error in this prediction. First, since a finite number of coupon tests are performed, the mean and standard deviation of the failure stress obtained through the coupon tests will be different from the actual mean and standard deviation.

In our earlier work [13], we neglected the effect of coupon tests and assumed the initial distribution of the mean failure stress $f^{i n i}\left(\bar{\sigma}_{f}\right)$ uniform within the bounds $b_{e}$ as

$$
f^{i n i}\left(\bar{\sigma}_{f}\right)=\left\{\begin{array}{cc}
\frac{1}{2 b_{e}\left(\sigma_{f}\right)_{\text {calc }}} & \text { if }\left|\frac{\bar{\sigma}_{f}}{\left(\sigma_{f}\right)_{\text {calc }}}-1\right| \leq b_{e} \\
0 & \text { othewise }
\end{array}\right.
$$

Then, the distribution of the mean failure stress is updated using the Bayesian technique with a given $\left(\sigma_{f}\right)_{1, \text { test }}$ as

$$
f^{\text {upd }}\left(\bar{\sigma}_{f}\right)=\frac{f_{1, \text { test }}\left(\bar{\sigma}_{f}\right) f^{i n i}\left(\bar{\sigma}_{f}\right)}{\int_{-\infty}^{\infty} f_{1, \text { test }}\left(\bar{\sigma}_{f}\right) f^{i n i}\left(\bar{\sigma}_{f}\right) d \bar{\sigma}_{f}}
$$

where $f_{1, \text { test }}\left(\bar{\sigma}_{f}\right)=\operatorname{Normal}\left(\left(\sigma_{f}\right)_{1, \text { test }} ; \bar{\sigma}_{f}, \operatorname{Std}\left(\sigma_{f}\right)\right)$ is the likelihood function reflecting possible variability of the first test result $\left(\sigma_{f}\right)_{1, \text { test }}$. Note that $f_{1, \text { test }}\left(\bar{\sigma}_{f}\right)$ is not a probability distribution in $\bar{\sigma}_{f}$; it is the conditional probability density of obtaining test result $\left(\sigma_{f}\right)_{1, \text { test }}$, given that the mean value of the failure stress is $\bar{\sigma}_{f}$. Subsequent tests are handled by the same equations, using the updated distribution, as the initial one.

If the Bayesian updating procedure defined above is used directly within an MCS loop for design thickness determination, the computational cost will be very high. In this paper, instead, the Bayesian updating is performed aside in a separate MCS loop. In this separate loop, we first simulate the coupon tests by drawing random samples for the mean and standard deviation of the calculated failure stress $\bar{\sigma}_{c f}$ and $\operatorname{Std}\left(\sigma_{c f}\right)$. Then, we simulate $n_{e}$ number of element tests, $\left(\sigma_{e f}\right)_{\text {test }}$. The element test results along with the mean and the standard deviation are used to define the likelihood function as $f_{1, \text { test }}\left(\bar{\sigma}_{f}\right)=\operatorname{Normal}\left(\left(\sigma_{e f}\right)_{1, \text { test }} ; \bar{\sigma}_{c f}, \operatorname{Std}\left(\sigma_{c f}\right)\right)$ in Eq. (A2). The initial distribution $f^{i n i}\left(\bar{\sigma}_{f}\right)$ in Eq. (A2) is uniformly distributed within some bounds as given in Eq. (A3). 


$$
f^{i n i}\left(\bar{\sigma}_{f}\right)=\left\{\begin{array}{cc}
\frac{1}{2 b_{e} \bar{\sigma}_{c f}} & \text { if }\left|\frac{\bar{\sigma}_{f}}{\bar{\sigma}_{c f}}-1\right| \leq b_{e} \\
0 & \text { othewise }
\end{array}\right.
$$

We found that applying the error bounds $b_{e}$ before the Bayesian updating or after the updating do not matter. Applying the error bounds before Bayesian updating means calculating the initial distribution $f^{\text {ini }}\left(\bar{\sigma}_{f}\right)$ from Eq. (A3) and then using Eq. (A2). To apply the error bounds after the Bayesian updating, however, we first assume very large error bounds $b_{e}$, calculate the initial distribution $f^{i n i}\left(\bar{\sigma}_{f}\right)$ from Eq. (3), and finally apply the error bounds $b_{e}$ to the distribution obtained using Eq. (A2).

Applying the error bounds after the Bayesian updating is more useful when we want to fit distributions (e.g., Johnson distribution) to the mean failure stress obtained through Bayesian updating. If we apply the error bounds at the beginning, the distribution after Eq. (A2) will be a truncated one and it will be difficult to fit a distribution with good fidelity. However, if we apply the error bounds at the end, the distribution after Eq. (A2) will be a continuous one and we will high likely fit a good distribution.

So the overall procedure is as follows. Within an MCS loop, we generate random mean and standard deviation values for the failure stress to be obtained through coupon tests. Then, we assume large error bounds to be used in Eq. (A3), simulate element tests and use Eq. (A2) to obtain the distribution of the mean failure stress. Then, we compute the four quartiles of the mean failure stress distribution. Finally, we compute the mean and standard deviations of the quartiles and we model these quartiles as normal distributions. Note that the quartiles are the values of failure stress for CDF values of [0.067, 0.309, 0.691, 0.933].

At first, we wanted to build response surfaces for the mean and standard deviation of the quartiles (after each element test) in terms of the number of coupon tests. Our numerical analysis revealed, on the other hand, that the number of coupon tests do not have a noticeable effect on the mean and standard deviation of the quartiles The variations of the of the mean and standard deviation of the first quartile of the mean failure stress (after the third element test) with number of coupon tests are depicted in Figure A1. Tables A1 through A3 presents the mean and standard deviation of the quartiles for the cases of 30,50 and 80 coupon tests, respectively. We see that the effect of coupon tests is buried under the numerical noise due to limited number of Monte Carlo sampling (10,000 samples).

Table A1. The mean and standard deviation of the quartiles of the mean failure stress after element tests if 30 coupon tests are performed.

\begin{tabular}{|c|c|c|c|c|c|c|c|c|}
\hline & \multicolumn{4}{|c|}{ Mean values of the quartiles $\left(\mathbf{Q}_{\mathbf{1 - 4}}\right)$} & \multicolumn{3}{c|}{ Standard deviation of the quartiles $\left(\mathbf{Q}_{\mathbf{1 - 4}}\right)$} \\
\hline & $\bar{Q}_{1}$ & $\bar{Q}_{2}$ & $\bar{Q}_{3}$ & $\bar{Q}_{4}$ & $\operatorname{std}\left(Q_{1}\right)$ & $\operatorname{std}\left(Q_{2}\right)$ & $\operatorname{std}\left(Q_{3}\right)$ & $\operatorname{std}\left(Q_{4}\right)$ \\
\hline test1 & 0.897 & 0.967 & 1.048 & 1.144 & 0.090 & 0.096 & 0.105 & 0.115 \\
\hline test2 & 0.924 & 0.975 & 1.032 & 1.096 & 0.076 & 0.080 & 0.084 & 0.090 \\
\hline test3 & 0.935 & 0.977 & 1.023 & 1.074 & 0.071 & 0.074 & 0.077 & 0.081 \\
\hline test4 & 0.943 & 0.980 & 1.020 & 1.063 & 0.068 & 0.070 & 0.073 & 0.076 \\
\hline test5 & 0.949 & 0.982 & 1.018 & 1.056 & 0.066 & 0.068 & 0.070 & 0.073 \\
\hline
\end{tabular}

Table A2. The mean and standard deviation of the quartiles of the mean failure stress after element tests if 50 coupon tests are performed.

\begin{tabular}{|c|c|c|c|c|c|c|c|c|}
\hline & \multicolumn{4}{|c|}{ Mean values of the quartiles $\left(\mathbf{Q}_{\mathbf{1 - 4}}\right)$} & \multicolumn{3}{|c|}{ Standard deviation of the quartiles $\left(\mathbf{Q}_{\mathbf{1 - 4}}\right)$} \\
\hline & $\bar{Q}_{1}$ & $\bar{Q}_{2}$ & $\bar{Q}_{3}$ & $\bar{Q}_{4}$ & $\operatorname{std}\left(Q_{1}\right)$ & $\operatorname{std}\left(Q_{2}\right)$ & $\operatorname{std}\left(Q_{3}\right)$ & $\operatorname{std}\left(Q_{4}\right)$ \\
\hline test1 & 0.901 & 0.970 & 1.051 & 1.146 & 0.092 & 0.098 & 0.106 & 0.115 \\
\hline test2 & 0.929 & 0.980 & 1.036 & 1.100 & 0.078 & 0.082 & 0.087 & 0.092 \\
\hline test3 & 0.940 & 0.982 & 1.028 & 1.078 & 0.073 & 0.076 & 0.080 & 0.084 \\
\hline test4 & 0.948 & 0.984 & 1.024 & 1.067 & 0.071 & 0.073 & 0.076 & 0.080 \\
\hline test5 & 0.952 & 0.986 & 1.021 & 1.059 & 0.068 & 0.070 & 0.072 & 0.075 \\
\hline
\end{tabular}

16

American Institute of Aeronautics and Astronautics 
Table A3. The mean and standard deviation of the quartiles of the mean failure stress after element tests if 80 coupon tests are performed.

\begin{tabular}{|c|c|c|c|c|c|c|c|c|}
\hline & \multicolumn{4}{|c|}{ Mean values of the quartiles $\left(\mathbf{Q}_{\mathbf{1 - 4}}\right)$} & \multicolumn{3}{c|}{ Standard deviation of the quartiles $\left(\mathbf{Q}_{\mathbf{1 - 4}}\right)$} \\
\hline & $\bar{Q}_{1}$ & $\bar{Q}_{2}$ & $\bar{Q}_{3}$ & $\bar{Q}_{4}$ & std $\left(Q_{1}\right)$ & $\operatorname{std}\left(Q_{2}\right)$ & std $\left(Q_{3}\right)$ & $\operatorname{std}\left(Q_{4}\right)$ \\
\hline test1 & 0.895 & 0.965 & 1.045 & 1.140 & 0.089 & 0.095 & 0.103 & 0.112 \\
\hline test2 & 0.922 & 0.972 & 1.029 & 1.092 & 0.074 & 0.078 & 0.082 & 0.087 \\
\hline test3 & 0.935 & 0.977 & 1.023 & 1.073 & 0.069 & 0.072 & 0.075 & 0.079 \\
\hline test4 & 0.943 & 0.979 & 1.019 & 1.062 & 0.065 & 0.068 & 0.070 & 0.074 \\
\hline test5 & 0.949 & 0.982 & 1.017 & 1.055 & 0.065 & 0.067 & 0.069 & 0.072 \\
\hline
\end{tabular}

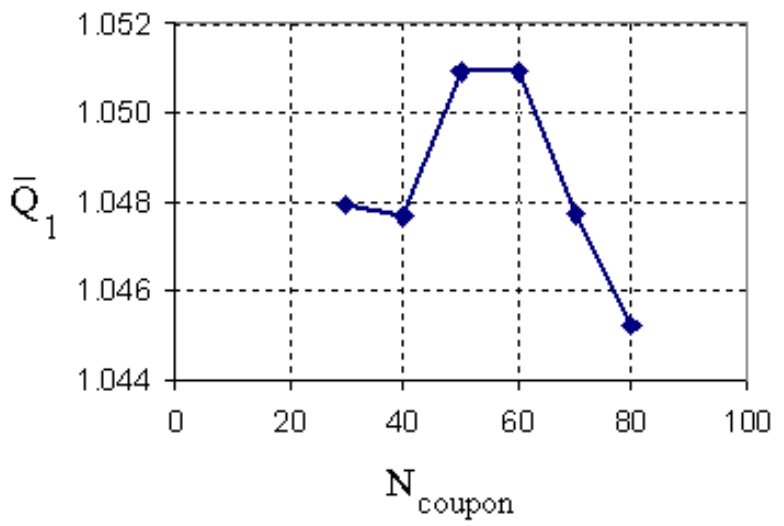

(a) mean value of the first quartile

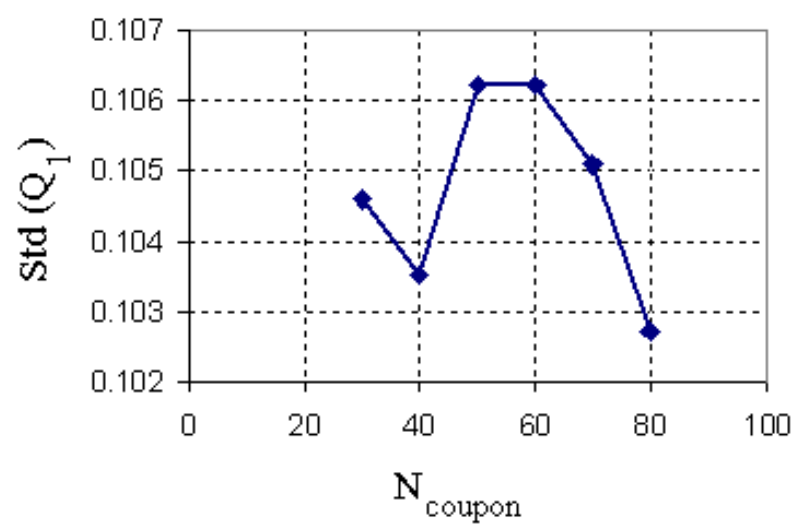

(a) standard deviation of the first quartile

Figure A1. Variation of the mean and standard deviation of the first quartile of the mean failure stress with number of coupon tests (after the third element test)

As noted earlier, the quartiles are assumed to have normal distributions. Figure A2 show the histograms of the first and second quartiles of the mean failure stress (after the third element test) obtained through MCS with 10,000 samples. We see that the quartiles do not exactly follow normal distributions.

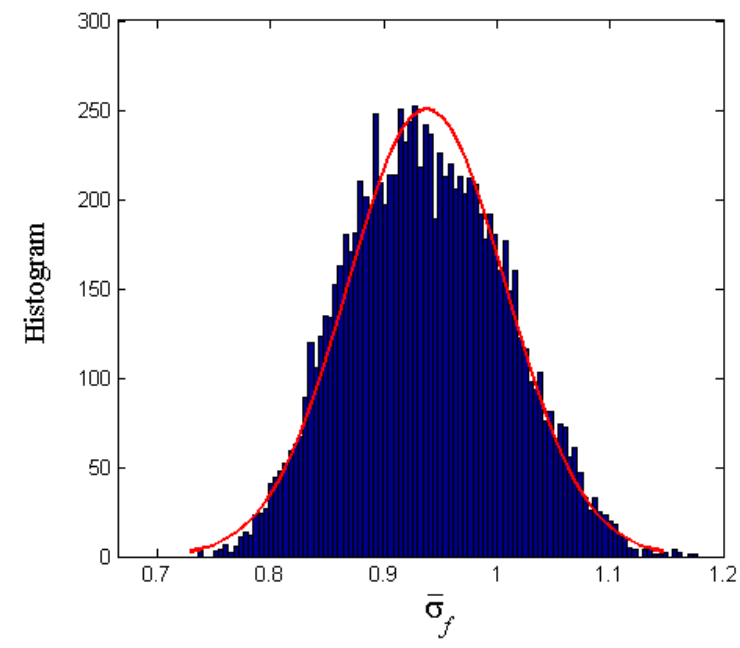

(a) histogram of the first quartile

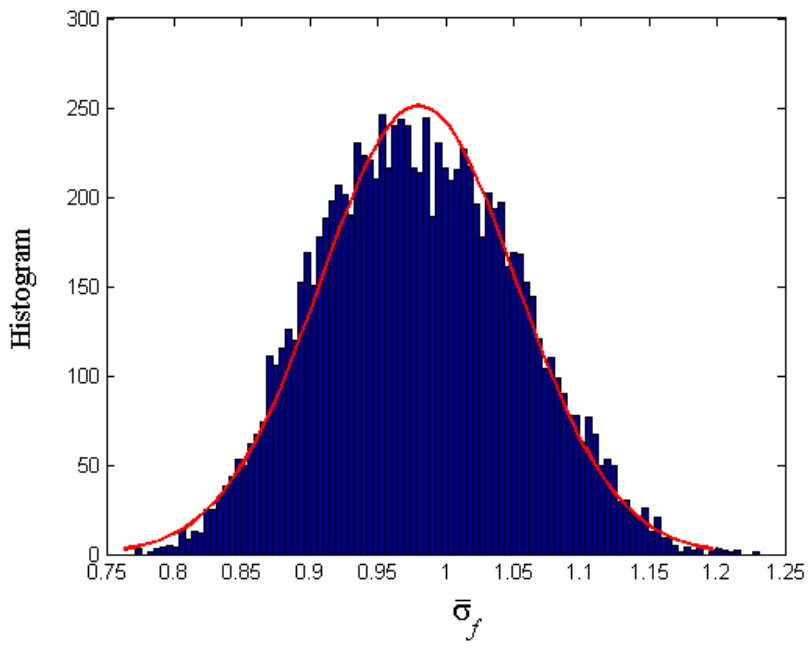

(a) histogram of the second quartile

Figure A2. Histograms of the first and the second quartiles of the mean failure stress (after the third element test). The continuous lines show the normal fits. 
The results obtained in this separate MCS loop are used in the main MCS loop for design thickness determination. The mean and standard deviations of the quartiles are used to fit a Johnson distribution to the mean failure stress. The error bounds $b_{e}$ are then applied to the Johnson distribution and random values from this distribution are drawn whenever element tests are simulated. Note also that the quartiles are strongly correlated to each other, so this correlation is also included in our analysis while random quartiles are generated in the main MCS loop for design thickness determination.

\section{Acknowledgments}

The authors gratefully acknowledge the support of the research by the NASA center and the U.S. Air Force, U.S. Air Force Research Laboratory, under award FA9550-07-1-0018 (Victor Giurgiutiu, Program Manager).

\section{References}

1. Zhu, H., Sankar, B.V., and Marrey, R.V., "Evaluation of Failure Criteria for Fiber Composites Using Finite Element Micromechanics," Journal of Composite Materials, Vol. 32, No. 8, 1998, pp. 766-782.

2. Federal Aviation Regulations, Part 25, Airworthiness Standards: Transport Category Airplanes, Sec. 25.303, Factor of Safety.

3. Federal Aviation Regulations, Part 25, Airworthiness Standards: Transport Category Airplanes, Sec. 25.613, Material Strength Properties and Material Design Values.

4. Composite Materials Handbook MIL-HDBK-17, "Guidelines for Property Testing of Composites," ASTM Publications, 2002.

5. Acar, E., Haftka, R.T., Sankar, B.V. and Qui, X., "Increasing Allowable Flight Loads by Improved Structural Modeling," AIAA Journal, Vol. 44, No. 2, 2006, pp. 376-381.

6. Qu, X., Haftka, R.T., Venkataraman, S., and Johnson, T.F., "Deterministic and Reliability-Based Optimization of Composite Laminates for Cryogenic Environments," AIAA Journal, Vol. 41, No. 10, 2003, pp. 2029-2036.

7. Acar, E., Haftka, R.T. and Johnson, T.F., "Tradeoff of Uncertainty Reduction Mechanisms for Reducing Structural Weight," Journal of Mechanical Design, Vol. 129, No. 3, 2007, pp. 266-274.

8. Federal Aviation Regulations, Part 25, Airworthiness Standards: Transport Category Airplanes, Sec. 25.307, Proof of Structure.

9. Oberkampf, W.L., Deland, S.M., Rutherford, B.M., Diegert, K.V., and Alvin, K.F., "Estimation of Total Uncertainty in Modeling and Simulation," Sandia Report, SAND2000-0824, 2000, Albuquerque, NM.

10. Oberkampf, W.L., Deland, S.M., Rutherford, B.M., Diegert, K.V., and Alvin, K.F., "Error and Uncertainty in Modeling and Simulation," Reliability Engineering and System Safety, Vol. 75, 2002, pp. 333-357.

11. Acar, E., Kale, A., Haftka, R.T. and Stroud, W.J., "Structural Safety Measures for Airplanes," Journal of Aircraft, Vol. 43, No. 1, 2006, pp. 30-38.

12. Acar, E., Kale, A., and Haftka, R.T., "Comparing Effectiveness of Measures that Improve Aircraft Structural Safety," Journal of Aerospace Engineering, Vol. 20, No. 3, 2007, pp. 186-199.

13. An, J., Acar, E., Haftka, R.T., Kim, N.H., Ifju, P.G., and Johnson, T.F., "Being Conservative with a Limited Number of Test Results," Journal of Aircraft, Vol. 45, No. 6, 2008, pp. 1969-1975.

14. Smarslok, B., Haftka, R.T., and Kim, N-H, "Comparison and Efficiency Analysis of Crude and Separable Monte Carlo Simulation Methods," 47th AIAA/ASME/ASCE/AHS/ASC Structures, Structural Dynamics and Materials Conference, Newport, RI, April 2006, AIAA Paper 2006-1632. 